\title{
Comparisons of FMM Implementations Employing Different Formulations and Iterative Solvers
}

\author{
Levent Gürel and Özgür Ergül \\ Department of Electrical and Electronics Engineering \\ Bilkent University \\ Ankara, Turkey \\ (lgurel@bilkent.edu.tr, ergul@ee.bilkent.edu.tr)
}

\section{Introduction}

Our ultimate goal is to solve very large numerical problems, which are obtained from mathematical formulations of real-life electromagnetic problems. In order to solve large problems, advances in both solution algorithms and computer hardware should be utilized. The fast multipole method (FMM) and its multi-level version, the multi-level fast multipole algorithm (MLFMA), are two of the preferred choices for the solution algorithm due to their reduced computational complexities and memory requirements. [1] As for the hardware, a parallel architecture is preferred due to its increased computing power. Consequently, this choice of hardware forces a parallel implementation of the MLFMA.

Implementation of the MLFMA requires the consideration of several parameters. Some of the high-level choices that need to be made are as follows:

- Integral-equation (IE) formulation: Electric-field IE (EFIE), magnetic-field IE (MFIE), or combined-field IE (CFIE), where CFIE $=\alpha \mathrm{EFIE}+(1-\alpha) \mathrm{MFIE}$.

- Iterative solver: Krylov-subspace methods such as conjugate gradient squared (CGS), biconjugate gradient (BiCG), biconjugate gradient stabilized (BiCGstab), generalized minimal residual (GMRES).

- Preconditioner: Near-field (NF) preconditioner, filtered NF preconditioner, blockdiagonal preconditioner, diagonal preconditioner, or no preconditioner. [2]

- Initial guess.

It is a substantial task to find the optimal or even a near-optimal combination of the above parameters, especially considering that the type of the problem to be solved is yet another parameter. It is important to note that a seemingly straightforward choice to simplify the formulation and to reduce the computational burden, such as using the EFIE with no preconditioner, backfires as a nonconvergent combination. As a remedy, one could use the CFIE formulation, which produces a better-conditioned system of equations, and a NF preconditioner to reduce the number of iterations. However, this combination has the disadvantages of CFIE not being suitable for some (open) geometries and the NF preconditioner not being easily parallelizable.

Therefore, the preferred combination of the parameters listed above is not trivially obvious and requires a careful investigation. In this paper, these parameters will be extensively investigated. For this purpose, a series of scattering problems of various sizes (at different frequencies) containing different numbers of unknowns will be used as a testbed. 


\section{Preconditioner Choices}

In order to investigate the effect of the preconditioners to the efficiency of the solution, variations in the other parameters are minimized and the solution is obtained with a number of different preconditioners. For this purpose, two scattering-from-sphere problems with 1302 and 8364 unknowns are formulated with the EFIE and solved using the CGS and BiCG iterative methods. The following preconditioners are employed for the solutions:

1. No preconditioner

2. Diagonal preconditioner: Only the diagonal elements of the impedance matrix are retained and used as the preconditioner.

3. Block-diagonal preconditioner: The diagonal partitions of the impedance matrix are retained and used as the preconditioner. The partitions are based on the clustering of the geometry.

4. NF preconditioner: The sparse matrix based on the NF interactions of the FMM is used as the preconditioner. This is a block matrix with the partitions formed in accordance with the clustering of the geometry.

5. Filtered NF preconditioner: Sparsity of the NF matrix is increased by eliminating the smaller elements of the matrix and by keeping those elements exceeding a preset threshhold. [3]

Figures 1 and 2 display the convergence characteristics (in terms of the residual versus the number of iterations) of the two scattering problems with 1302 and 8364 unknowns, respectively, with various preconditioners. We note that BiCG method consistently outperforms the CGS method in these results. We also note that the convergence is accelerated as the sparsity of the preconditioner matrix reduces. Even though this general behavior is expected, we note that the block-diagonal preconditioner constitutes an important exception to this behavior. The importance of this exception is due to the suitability of this preconditioner for parallel programming.

\section{Integral Equation Formulation}

Figure 3 compares the convergence characteristics of four different scattering-fromsphere problems with different numbers of unknowns. The three formulations compared in these results are EFIE, MFIE, and CFIE with $\alpha=0.2$. The iterative solution is obtained with the CGS method utilizing a block-diagonal preconditioner. Failure of convergence for the EFIE formulation is expected from the results presented in Figures 1 and 2. The same behavior is observed for the EFIE in Figure 3, too. However, we note that the MFIE and CFIE formulations converge rapidly with the block-diagonal preconditioner. Furthermore, we note that the number of iterations required for the convergence of the CFIE is almost independed of the number of unknowns, whereas the number of iterations required by the MFIE increases significantly for larger problems. This trend is tested by solving much larger problems with 36,862 and 132,003 unknowns. Both problems are solved using an MLFMA implementation employing the CFIE formulation, CGS solver, and block-diagonal 


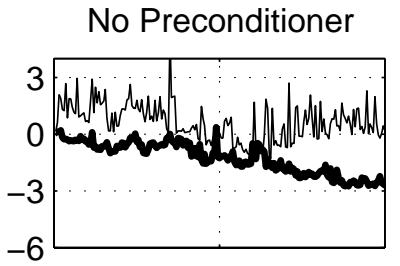

Diagonal (1302 elements)

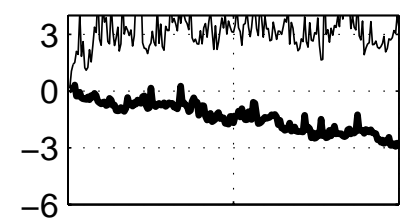

Block Diagonal (46416 elements)
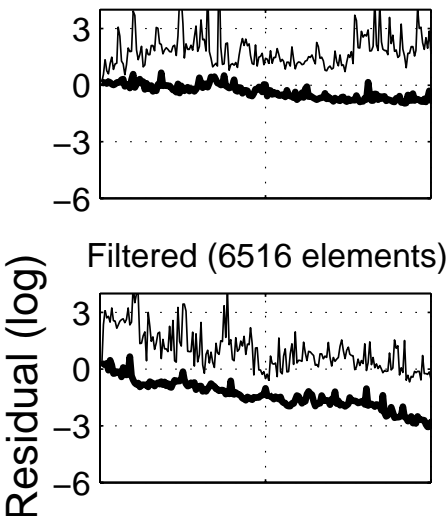

Filtered (28992 elements)

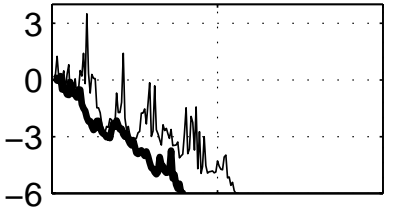

Filtered (126754 elements)

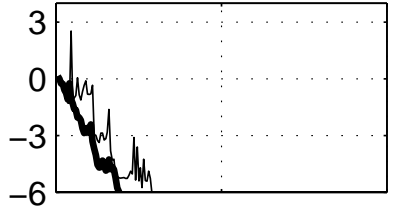

Filtered (1268700 elements)

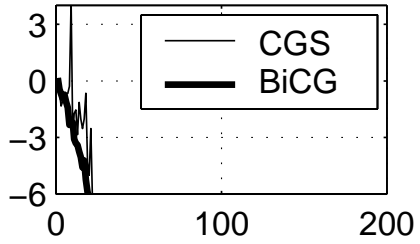

Number of Iterations

Figure 1. Convergence characterictics of a 1302 unknown scattering problem formulated with the EFIE and solved with BiCG and CGS employing various preconditioners.
No Preconditioner

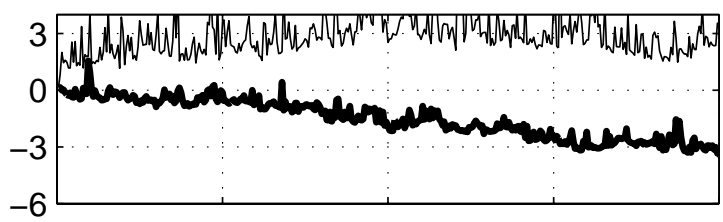

Diagonal (8364 elements)

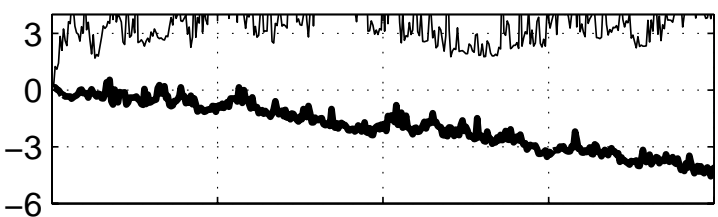

Block Diagonal (770438 elements)

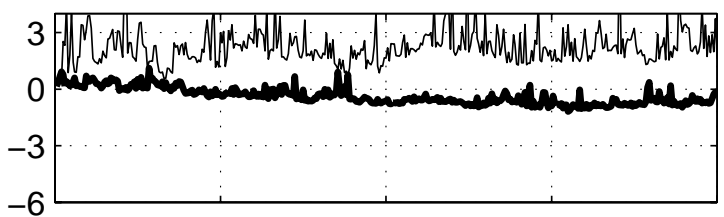

Filtered (63300 elements)

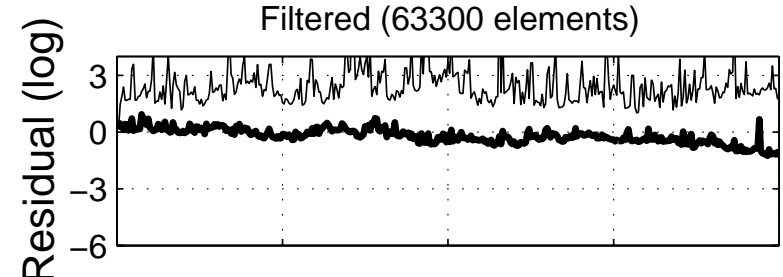

Filtered (181864 elements)

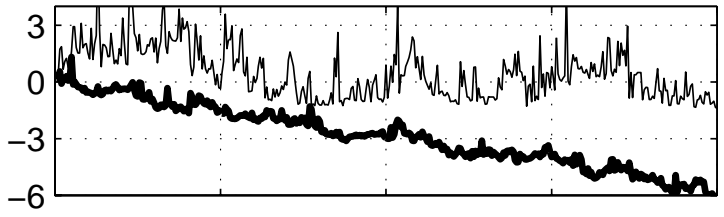

Filtered (245344 elements)

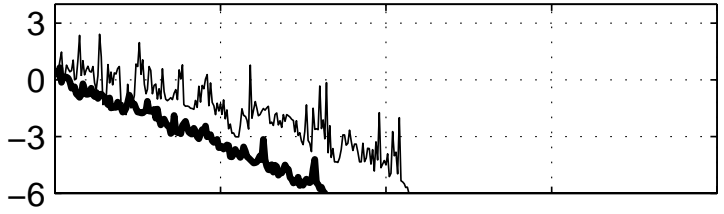

Filtered (2408632 elements)

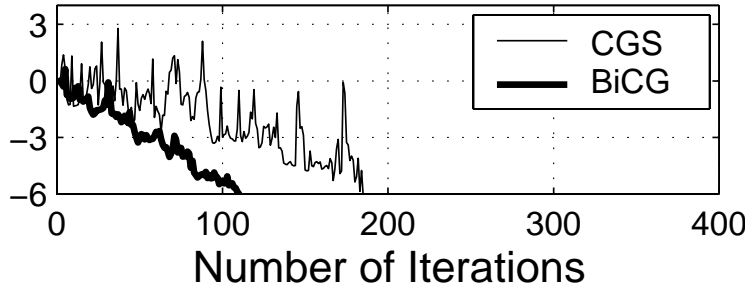

Figure 2. Convergence characterictics of a 8364 unknown scattering problem formulated with the EFIE and solved with BiCG and CGS employing various preconditioners. 
preconditioner. In these two problems, the residual error is reduced to $10^{-6}$ in 20 and 24 iterations, respectively.
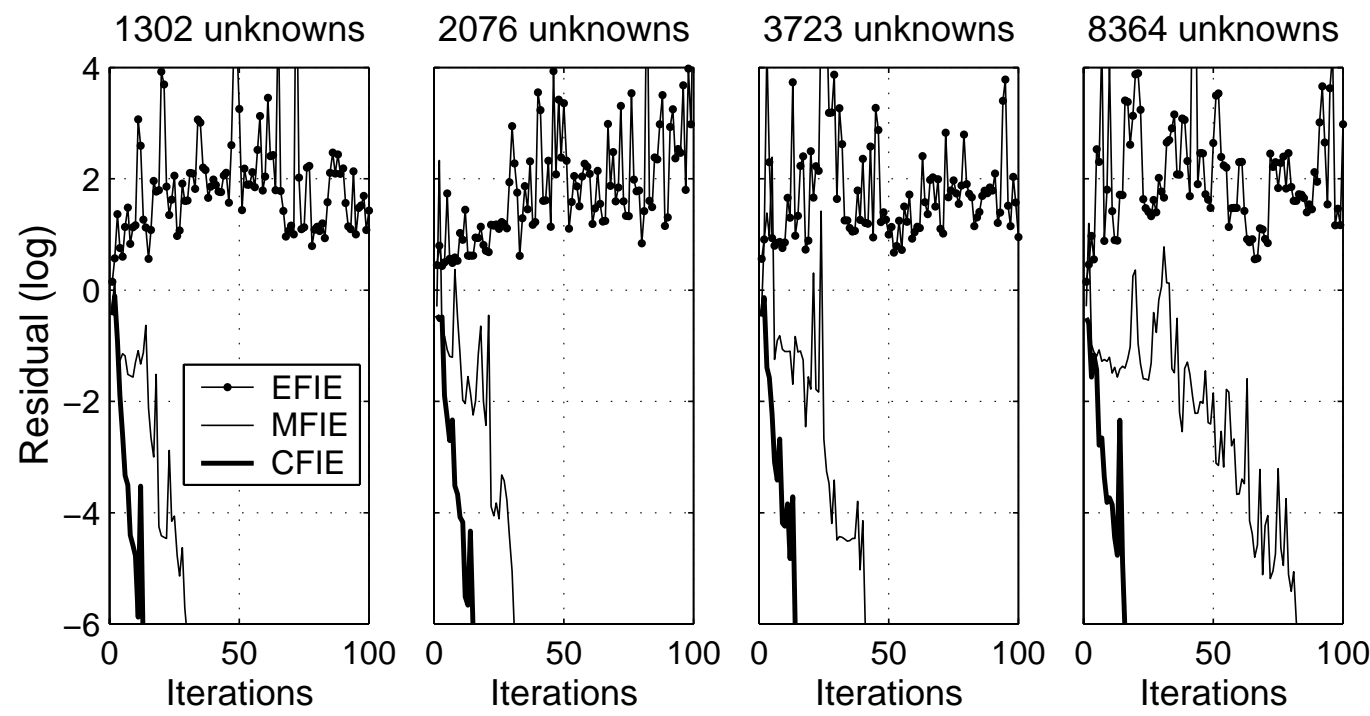

Figure 3. Comparison of the convergence characteristics of the EFIE, MFIE, and CFIE formulations of scattering problems of various sizes solved with CGS and block-diagonal preconditioner.

\section{Conclusions}

We compare the performances of various single-level FMM and MLFMA implementations. Choices of the integral-equation formulations, iterative solvers, preconditioners, and initial guesses are considered as parameters in order to determine the most favorable combination for a given type pf the problem.

\section{Acknowledgements}

This work was supported by the Turkish Academy of Sciences in the framework of the Young Scientist Award Program (LG/TUBA-GEBIP/2002-1-12) and by Bilkent University under research fund EE-01-01.

\section{References}

[1] W. C. Chew, J.-M. Jin, E. Michielssen, and J. Song, Eds., Fast and Efficient Algorithms in Computational Electromagnetics. Boston: Artech House, 2001.

[2] J. Song, C. C. Lu, and W. C. Chew, "Multilevel fast multipole algorithm for electromagnetic scattering by large complex objects," IEEE Trans. Antennas Propagat., vol. 45, no. 10, pp. 1488-1493, Oct 1997.

[3] L. Gürel and H. Bağc1, "Fast-multipole-method solution of the electromagnetic scattering from a stealth geometry," Bilkent University Research Report, 2002. 